\title{
PERFIL PSICOLÓGICO DE PACIENTES COM ANOREXIA E BULIMIA NERVOSAS: A ÓTICA DO PSICODIAGNÓSTICO
}

\author{
PSYCHOLOGICAL PROFILE OF PATIENTS WHO SUFFER FROM ANOREXIA NERVOSA \\ AND BULIMIA NERVOSA: PSYCHOLOGICAL ASSESSMENT POINT OF VIEW
}

Érika Arantes de Oliveira ${ }^{1}$, Manoel Antônio dos Santos²

\begin{abstract}
${ }^{1}$ Psicóloga. ${ }^{2}$ Docente. Bolsista de Produtividade em Pesquisa do CNPq. Departamento de Psicologia e Educação. Faculdade de Filosofia, Ciências e Letras de Ribeirão Preto - USP.

Correspondência: Érika Arantes de Oliveira. Rua Machado de Assis, 433 - Vila Tibério. CEP 14050-490 - Ribeirão Preto-SP.

E-mail: erikaao@ffclrp.usp.br / Fone: 1636023645
\end{abstract}

Oliveira EA, Santos MA. Perfil psicológico de pacientes com anorexia e bulimia nervosas: a ótica do psicodiagnóstico. Medicina (Ribeirão Preto) 2006; 39 (3): 353-60.

RESUMO: O presente trabalho tem por objetivo apresentar o perfil psicológico dos pacientes portadores de anorexia e bulimia nervosas, visando fornecer subsídios para o planejamento de intervenções a serem implementadas por equipe interdisciplinar. Foram realizadas 29 avaliações psicodiagnósticas, compreendendo 24 mulheres e cinco homens. Os instrumentos utilizados foram: técnicas projetivas, teste de nível intelectual e escalas padronizadas, complementados por entrevista clínica. Como resultados pôde-se constatar, mediante as entrevistas, a negação do adoecimento e, conseqüentemente, o não reconhecimento da necessidade de tratamento. Quanto aos resultados fornecidos pelos demais instrumentos observou-se que, de um modo geral, os recursos intelectuais encontram-se dentro da média esperada, porém evidencia-se marcado comprometimento emocional dos pacientes portadores de transtornos alimentares, embora esteja preservada a possibilidade de vinculação afetiva, o que atesta a necessidade de suporte psicoterapêutico.

Descritores: Anorexia Nervosa. Bulimia Nervosa. Avaliação Psicológica. Transtornos da Alimentação.

\section{1 - INTRODUÇÃ̃O}

Os transtornos alimentares são quadros psiquiátricos caracterizados por uma grave perturbação do comportamento alimentar, sendo a anorexia e a bulimia nervosas os dois tipos principais ${ }^{1}$.

A anorexia nervosa pode ser definida como uma recusa sistemática em manter o peso no mínimo normal adequado à idade e altura, acompanhada de uma perturbação no modo como o indivíduo vivência seu peso e sua forma física. Já a bulimia nervosa caracteriza-se por episódios de comer compulsivo, associados a sentimento de descontrole sobre o comportamento alimentar. Nos dois casos o peso e o formato corporal exercem marcada influência na determinação da auto-estima dos pacientes ${ }^{1}$, que via de regra encontra-se rebaixada.

Acredita-se atualmente que exista uma etiopatogenia multifatorial, com hipóteses da influência combinada da dinâmica familiar, do meio cultural e de aspectos da personalidade do indivíduo como fatores concorrentes para a predisposição, instalação e manutenção dos distúrbios. 


\section{1- Dinâmica familiar}

A influência do ambiente familiar sobre os transtornos alimentares é uma das dimensões mais valorizadas desde que essas enfermidades foram descritas, sendo que fatores familiares podem contribuir tanto para o desencadeamento como para a manutenção do transtorno ${ }^{2}$.

Alguns autores descrevem a existência de um padrão de confusão das fronteiras que definem os subsistemas familiares (parental, filial). Esse padrão seria marcado pela ausência generalizada de limites entre gerações e pessoas dos diferentes subsistemas, faltando o senso de identidade e individualidade dos membros familiares, que apresentariam padrões inadequados de relacionamento interpessoal. Tais famílias exibiriam uma harmonia aparente que, na verdade, ocultaria graves conflitos subjacentes ${ }^{3,4}$.

\section{2- Meio sociocultural}

No mundo contemporâneo as imagens do corpo esbelto são cada vez mais cultuadas pelos meios de comunicação de massa, geralmente associadas pelo marketing à obtenção de sucesso e prestígio em uma sociedade de consumo. Esse contexto macrossocial proporcionaria novas formas de vivenciar subjetivamente a corporeidade e de se relacionar com o corpo, contribuindo, juntamente com outros fatores, para o cultivo de um caldo de cultura que favoreceria a incidência dos transtornos alimentares ${ }^{5}$.

Isso não significa endossar a tese de uma produção eminentemente social desses sintomas, nem validar a existência de uma relação linear do tipo causa-efeito, uma vez que durante algum tempo acreditou-se que a anorexia incidiria apenas em mulheres com bom nível socioeconômico e educacional e oriundas de países desenvolvidos de cultura ocidental, com oferta abundante de alimentos. Contudo, estudos recentes desmentem categoricamente essa crença e demonstram que os transtornos da conduta alimentar não atingem somente indivíduos com esse perfil restrito ${ }^{5}$.

\section{3- Perfil de personalidade}

Quanto aos aspectos de personalidade envolvidos, alguns autores acreditam que o transtorno alimentar corresponderia a uma apresentação atípica de um transtorno afetivo ou de um transtorno obsessivo-compulsivo ${ }^{4}$.

A baixa auto-estima, bem como a distorção da imagem corporal, estariam relacionadas com a origem da anorexia nervosa. Pacientes com anorexia do subtipo purgativo tenderiam a ser mais impulsivos do que os restritivos, que seriam mais perfeccionistas e obsessivos. Como características psicológicas salientes esses pacientes tendem a apresentar: baixa auto-estima, sentimento de desesperança, desenvolvimento insatisfatório da identidade, tendência a buscar aprovação externa, hipersensibilidade à crítica e conflitos referentes às questões de autonomia versus independência ${ }^{6}$.

Pacientes com bulimia nervosa apresentariam pensamentos e emoções desadaptativas, auto-estima flutuante, sendo comum encontrar aqueles que apresentam atitudes caóticas, não somente no tocante aos hábitos alimentares, mas também em outros aspectos da vida, como os estudos, a vida profissional e as relações amorosas. Para alguns autores os principais aspectos emocionais dos pacientes com anorexia nervosa seriam: baixa auto-estima; ansiedade alta, perfeccionismo, pensamento do tipo "tudo ou nada", incapacidade de encontrar formas de satisfação, alta exigência e incapacidade de ser feliz ${ }^{6}$.

\section{4- O processo de avaliação psicodiagnóstica}

Levando em consideração a complexidade dos fatores envolvidos na origem e na manutenção dos quadros de transtornos alimentares, como demonstrado acima, o Grupo de Assistência em Transtornos Alimentares (GRATA) do Ambulatório de Nutrologia do Hospital das Clínicas da Faculdade de Medicina de Ribeirão Preto da Universidade de São Paulo (HC-FMRP-USP) realiza uma avaliação psicodiagnóstica dos seus pacientes, na tentativa de melhor compreender seu funcionamento psicodinâmico.

O processo psicodiagnóstico pode ser caracterizado como uma situação bi-pessoal (psicólogo-paciente), de duração limitada, cujo objetivo é a descrição e compreensão da personalidade do paciente, utilizando-se para alcançar este objetivo as seguintes técnicas: entrevistas semidirigidas, técnicas projetivas e entrevistas de devolução. O psicólogo, ao planejar uma bateria de testes, deve escolher os que captem o maior número de condutas e em seus pormenores, estabelecendo a melhor seqüência em que os instrumentos de avaliação psicológica deverão ser aplicados ${ }^{7}$.

O presente trabalho tem por objetivo apresentar aspectos do funcionamento psicodinâmico dos pacientes portadores de anorexia e bulimia nervosas, submetidos à avaliação psicodiagnóstica, tanto no tocante aos aspectos emocionais, como no que concerne 
aos aspectos intelectuais que compõem a personalidade, com vistas a fornecer subsídios para o planejamento de intervenções terapêuticas por parte da equipe interdisciplinar.

\section{2 - MATERIAL E MÉTODOS}

\section{1- Participantes}

Foram realizadas 29 avaliações psicodiagnósticas, com 24 pacientes do sexo feminino e cinco do sexo masculino, vinculados ao Grupo de Assistência em Transtornos Alimentares (GRATA) do Hospital das Clínicas da Faculdade de Medicina de Ribeirão Preto da Universidade de São Paulo (HC-FMRP-USP). A faixa etária variou dos 14 aos 33 anos de idade e a maioria dos pacientes examinados era solteira. Em relação ao diagnóstico a maior parte era portadora de bulimia nervosa, seguidos de pacientes com anorexia nervosa do subtipo purgativo.

Tabela I: Caracterização da amostra de pacientes com transtornos alimentares em função do sexo, idade, estado civil e categoria diagnóstica. $(\mathrm{n}=29)$

\begin{tabular}{|l|c|c|}
\hline Variáveis & Freqüiência & $\%$ \\
\hline Sexo & & \\
\hline Masculino & 05 & 17 \\
\hline $\begin{array}{l}\text { Feminino } \\
\text { Total }\end{array}$ & 24 & 83 \\
\hline Idade & 29 & 100 \\
\hline $14-18$ & 17 & \\
\hline $19-23$ & 12 & 49 \\
\hline Estado Civil & & \\
\hline Casado & 03 & 10 \\
\hline Solteiro & 26 & 90 \\
\hline $\begin{array}{l}\text { Diagnóstico } \\
\text { Anorexia Nervosa }\end{array}$ Bulimia Nervosa & 11 & 38 \\
\hline
\end{tabular}

\section{2- Instrumentos e materiais}

Como instrumentos foi utilizada uma bateria de técnicas projetivas, teste de nível intelectual e escalas padronizadas (Escala de Atitudes Alimentares - EAT26 e Escala de Autoconceito de Janis e Field, revisada por Eagly). Essa bateria foi complementada por uma entrevista clínica, delineada especialmente para atender aos objetivos da avaliação.

\section{3- Entrevista clínica}

A entrevista pode ser considerada um dos principais instrumentos de investigação do funcionamento psicológico de um indivíduo, já que permite ao investigador recolher dados descritivos na própria linguagem do sujeito, considerando que esta corresponde a um sistema de sinais com função indicativa, comunicativa, expressiva e conotativa. Nesse sentido, oferece a possibilidade de o investigador desenvolver intuitivamente uma idéia aproximativa sobre o modo como o sujeito interpreta 0 mundo ${ }^{8}$, de acordo com sua história de vida, seu contexto cultural, seus valores referenciais, suas construções e atribuições de significados.

A entrevista seguiu um roteiro estruturado, abarcando os seguintes tópicos: Dados de identificação do paciente; Infância, adolescência e vida adulta; Relacionamentos na esfera sócio-familiar; Processo de adoecimento; Tratamentos anteriores; Trabalho/estudos; Eventos estressores e Planos futuros.

\section{Teste de Nível Intelectual}

\section{4- Matrizes Progressivas de Raven - Escala Geral}

Trata-se de um instrumento válido para avaliar a capacidade de pensamento lógico e trabalho intelectual preciso ${ }^{9}$. Esse teste é composto por cinco subtestes, constituídos por 12 itens cada um, organizados em ordem crescente de dificuldade. Os pacientes freqüentemente apontam a semelhança desse instrumento com testes utilizados na avaliação psicotécnica, expressando em geral desconforto com a realização do mesmo.

\section{Técnicas Projetivas}

\section{5- Teste de Apercepção Temática (TAT)}

O TAT é um método projetivo que visa detectar os conflitos e tendências da personalidade, assim como revelar dinamismos, atitudes e frustrações do indivíduo ${ }^{10}$. São apresentadas para o paciente pranchas com algumas gravuras em preto e branco, contendo cenas em sua maioria estruturadas, geralmente envolvendo personagens humanos. É solicitado que o sujeito conte uma história a partir do que percebe do material, contendo início, meio e fim. Para o presente estudo foram escolhidas 11 pranchas $(1,2,3 \mathrm{RH}, 4,7 \mathrm{RM}, 8 \mathrm{RM}, 11$, 12RM, 16, 18MF, 20) considerando-se ainda a necessária adequação do estímulo dependendo do gênero do examinando. 


\section{6- Questionário Desiderativo}

O Questionário Desiderativo é uma técnica projetiva verbal que informa sobre características de personalidade do paciente, sua bagagem defensiva, conflitos básicos, desenvolvimento cognitivo, auto-imagem, identidade sexual, dentre outros aspectos ${ }^{11}$.

\section{5- Exame de Rorschach}

O Rorschach ocupa posição privilegiada dentre as técnicas de avaliação de personalidade, sendo uma das mais utilizadas em diversos países. Suas possibilidades interpretativas abrem caminho para a compreensão acurada do ser humano em suas motivações profundas ${ }^{12}$. Também é uma técnica de cartões ou pranchas, mas diferentemente do TAT não apresenta estímulo determinado, mas amorfo, uma vez que as pranchas são constituídas de borrões de tinta de formas indefinidas, colocando o sujeito em contato com um material menos estruturado.

\section{6- Teste das Pirâmides Coloridas de Pfister}

O Teste das Pirâmides Coloridas de Pfister é uma técnica considerada válida e precisa (fidedigna), empregada para a compreensão do funcionamento afetivo-emocional do sujeito ${ }^{13}$. Sua maior vantagem repousa na economia de recursos, rapidez de execução e facilidade de compreensão da tarefa proposta, além do aspecto lúdico inerente à técnica, uma vez que o paciente trabalha com a escolha de cores, manipulando quadrículos de papel colorido em uma ampla gama de cores.

\section{7- Teste da Casa-Árvore-Pessoa (House-Tree- Person Test - HTP)}

O HTP tem o objetivo de fornecer uma visão panorâmica da personalidade do sujeito, em especial das suas interações com o mundo exterior ${ }^{14}$. Trata-se de um teste projetivo gráfico no qual o paciente é convidado a realizar determinados desenhos temáticos (casa, árvore, pessoa). Concluída a parte gráfica, o profissional realiza um inquérito verbal, utilizando-se para tanto de um roteiro padronizado de questões, para complementação das informações obtidas e exploração das fantasias inconscientes projetadas nos desenhos.

\section{3 - PROCEDIMENTO DE COLETA DE DADOS}

O estudo contou com a aprovação do Comitê de Ética em Pesquisa do HC-FMRP-USP. As avaliações foram realizadas individualmente em uma sala reservada e que oferecia condições adequadas de conforto e privacidade, junto à Clínica Psicológica do Centro de Psicologia Aplicada (CPA) da Faculdade de Filosofia, Ciências e Letras de Ribeirão Preto da Universidade de São Paulo (FFCLRP-USP). O processo foi desenvolvido ao longo de cinco encontros de aproximadamente uma hora de duração cada, complementado por um último encontro, dedicado à realização da entrevista devolutiva.

\section{1- Primeiro encontro}

Reservado para a explicitação da natureza da proposta, seus objetivos e modus operandi. Durante o estabelecimento do contrato de trabalho era enfatizado o caráter voluntário da participação do paciente. Uma vez obtida a anuência explícita do participante, mediante a assinatura do Termo de Consentimento Livre e Esclarecido, era realizada a entrevista clínica. Essa entrevista teve duração média de uma hora.

As entrevistas não foram audiogravadas. A psicóloga realizava algumas anotações durante o encontro e efetuava a transcrição do conteúdo verbal imediatamente após o término das entrevistas.

\section{2- Segundo encontro}

Nesse encontro era aplicado o Exame de Rorschach. A aplicação dessa técnica demandava cerca de 50 minutos, sendo que a aplicação mais rápida teve duração de 20 minutos e a mais longa, de 52 minutos.

\section{3- Terceiro encontro}

Foram aplicados nesse encontro o HTP e o Teste de Pirâmides Coloridas de Pfister. O primeiro despendeu cerca de 45 minutos em média para a sua realização na íntegra e o segundo, aproximadamente 15 minutos.

\section{4- Quarto encontro}

Nesse encontro eram aplicadas as Matrizes Coloridas de Raven. Sua aplicação em geral consumiu de 45 a 50 minutos.

\section{5- Quinto encontro}

Nesse encontro eram aplicados o Questionário Desiderativo e o TAT. Nos primeiros os pacientes necessitavam de cerca de 20 minutos para concluírem a tarefa e, no segundo, de 40 a 45 minutos.

\section{6- Sexto encontro}

O último encontro era destinado à entrevista devolutiva dos resultados para os pacientes, culminando com a proposta de conduta terapêutica. 


\section{4- PROCEDIMENTO DE ANÁliSE DOS DADOS}

Os dados obtidos por meio da aplicação dos instrumentos avaliativos foram cotados segundo as recomendações preconizadas pela literatura consagrada a cada técnica.

A entrevista foi analisada qualitativamente, mediante uma análise de conteúdo categorial, visando identificar as concepções, crenças, valores, motivações e atitudes dos entrevistados. Para efetuar a sistematização do material coletado foram seguidas as três etapas do processo de análise de conteúdo, a saber: pré-análise (organização do material e sistematização das idéias cruciais), descrição analítica (categorização dos dados em unidades de registros) e interpretação referencial (tratamento dos dados e interpretações) $)^{8,15}$.

Os resultados foram interpretados segundo a abordagem psicodinâmica que, do ponto de vista teórico-metodológico, fundamenta a maior parte dos instrumentos empregados no processo psicodiagnóstico.

\section{5- RESULTADOS}

\section{1- Organização intelectual e pragmatismo}

\section{Nível intelectual}

Observou-se que, de um modo geral, a capacidade intelectual encontra-se dentro da média esperada, ou seja, os resultados, expressos em percentis, situam-se dentro do intervalo definido pelos valores normativos disponíveis pelo teste utilizado.

\section{Capacidade produtiva}

Apesar de não haver prejuízo do potencial intelectual, notou-se um comprometimento da capacidade produtiva devido à interferência de fatores indicativos de imaturidade emocional. Além disso, constatou-se rebaixamento da síndrome de dinamismo, indicando um comprometimento da tríade ação-realização-produtividade.

Desse modo, apesar de apresentarem a função lógica preservada, a capacidade produtiva encontra-se prejudicada pela afetividade.

Parece que eu nunca mais vou conseguir fazer nada... E eu fico procurando a Ana (nome próprio) que eu era. (Feminino, 19 anos, bulimia nervosa)

\section{2- Organização afetiva}

\section{Mecanismos de controle da impulsividade}

Em relação aos aspectos afetivos, detecta-se uma falha nos mecanismos de controle da impulsividade, desencadeando inabilidade nas expressões afetivas, reações de cólera e descargas impulsivas.

Quando vi, já foi... (masculino, 18 anos, bulimia nervosa)

Tais descargas podem ser voltadas tanto para o meio externo como para o próprio organismo (autoagressividade).

\section{Relação consigo mesmo e auto-imagem}

A auto-imagem é pobremente estruturada, oferecendo uma sustentação precária para o sentimento de identidade pessoal. Reações afetivas hostis são amplamente dominantes, quando comparadas com as experiências de gratificação das necessidades amorosas. A projeção da hostilidade no ambiente é uma tendência marcante, dado ao baixo nível de tolerância aos conflitos e pouca continência aos afetos potencialmente disruptivos. Na tentativa de proteger-se da hetero-agressividade, os pacientes tendem, defensivamente, ao isolamento social e, na presença de situações de maior estresse ambiental, tornam-se suscetíveis a manifestações auto-agressivas, apresentando sinais de ansiedade introjetada, inquietações internas e tendência ao auto-ataque.

Em qualquer lugar que eu chego eu acho que sou a mais feia, a mais mal vestida. (feminino, 19 anos, anorexia nervosa)

A relação consigo também é marcada por conflito, sendo comum a baixa auto-estima, combinada com acentuada timidez, tendendo à introversão patológica.

\section{Relacionamentos com as figuras parentais}

$\mathrm{O}$ relacionamento com as figuras parentais, em especial no tocante à imago materna, é vivenciado de forma ambivalente, oscilando entre a extrema dependência e o desejo de completa liberdade e autonomia. Os sentimentos são vivenciados muito intensamente, tanto de ódio como de amor. Além disso, são expressos com o filtro de experiências do tipo "tudo ou nada", sem nuances e gradações, desencadeando reações explosivas.

Às vezes eu sei que tudo que ela (mãe) faz é para o meu bem, mas às vezes tenho um ódio dela! (feminino, 18 anos, bulimia nervosa) 


\section{Relacionamentos interpessoais}

Como a organização do relacionamento com as figuras estruturantes do psiquismo (imagos parentais) é precária, a relação com o outro também apareceu prejudicada, sendo que os relacionamentos profundos são considerados ameaçadores e tenazmente evitados. Os relacionamentos superficiais são marcados pela baixa espontaneidade e intensa insegurança. São freqüentes os sentimentos de solidão, isolamento, incompreensão, desamparo e desesperança.

Ando muito nervoso, as pessoas se afastam de mim... Ou eu que me afasto das pessoas? (masculino, 17 anos, bulimia nervosa)

\section{Sentimentos de desamparo e solidão}

Transpareceram no discurso dos pacientes sentimentos de desamparo e solidão extrema, bem como a não-confiança na capacidade de compreensão do outro e a "sensação de ser diferente no mundo".

Eu sou diferente, às vezes me sinto como um extraterrestre. (feminino, 25 anos, bulimia nervosa)

Alguns pacientes demonstraram ter uma consciência dolorosa acerca de seu empobrecimento afetivo, ressentindo-se da falta de uma bússola empática para se orientar no mundo das relações afetivas.

Eu me sinto muito sozinho no mundo... Às vezes acho que, se eu me matar, ninguém vai sentir a minha falta. (masculino, 17 anos, bulimia nervosa)

\section{3- $O$ adoecimento}

\section{Doença: ganho secundário}

Apareceu no discurso da maioria dos pacientes avaliados a questão do ganho secundário advindo do adoecimento, em especial a maior aproximação com os pais e a obtenção de um lugar de destaque na família, ainda que pelo viés negativo.

Antes eu achava que a minha mãe preferia meu irmão, agora (depois do transtorno alimentar) inverteu... Sempre me defende. (feminino, 16 anos, bulimia nervosa)

Os sintomas suscitam reações extremas e paroxísticas, que oscilam desde a absoluta indulgência até uma rejeição escancarada por parte de alguns familiares.

Meus irmãos não acreditam que eu tenho uma doença, acham que eu como escondido de todos. Dizem que é tudo frescura, que eu faço isso só pra chamar a atenção dos meus pais. (feminino, 26 anos, anorexia nervosa)

\section{Negação do adoecimento}

Não sei se sou mesmo doente... Hoje em dia todo mundo quer emagrecer a qualquer custo. (feminino, 23 anos, anorexia nervosa)

Os pacientes inicialmente "negam" a condição de adoecimento, alegando tratar-se de um estilo de vida, uma opção que assumiram conscientemente para lidar com suas preocupações com a forma física, uma estratégia eficaz para a manutenção do peso.

Acho que é um jeito que arrumei de manter o meu peso, não acho que eu seja doente. (masculino, 17 anos, bulimia nervosa)

\section{Mudanças no discurso}

No decorrer do processo de avaliação psicodiagnóstica alguns pacientes apresentaram uma modificação no discurso referente à sua percepção do adoecimento.

Eu nunca quis aceitar que tenho anorexia, queria pensar que era desnutrição. (feminino, 23 anos, anorexia nervosa)

\section{4- Atitude frente ao tratamento}

\section{Negação da necessidade de tratamento}

Eu fico ouvindo as moças (profissionais da equipe) falar e acho que ali não é o meu lugar... Tô pegando vaga de quem precisa de verdade. (feminino, 17 anos, bulimia nervosa)

Da mesma forma que negam - e até porque negam - o próprio adoecimento, revelam uma atitude de desvalorização e recusa do tratamento, incluindo a necessidade de participação na avaliação psicológica.

Venho para agradar a minha mãe. (feminino, 17 anos, anorexia nervosa)

\section{Mudanças no discurso}

Ao término do processo de avaliação psicodiagnóstica, alguns pacientes já apresentam uma postura mais reflexiva em relação ao adoecimento e ao tratamento:

Às vezes eu acho que os outros estão loucos... Depois penso que eu posso estar doente! Será que posso pensar que TODOS estão mentindo para mim? Nessas horas penso que preciso de ajuda. (feminino, 30 anos, bulimia nervosa)

\section{5- A Entrevista devolutiva}

A entrevista devolutiva era um momento eleito não só para fornecer as informações oriundas da 
avaliação e sugerir opções de encaminhamento - em geral para acompanhamento psicológico, como também um momento para efetuar uma espécie de "balanço" do trabalho de compreensão do psicodinamismo do paciente até então realizado.

No decorrer desse encontro que marcava o desfecho do processo ficava evidente a mudança do padrão de relacionamento estabelecido com a psicóloga-avaliadora. Inicialmente os pacientes colocavam-se de forma muito distanciada: Venho aqui porque a médica falou para eu vir, evoluindo para momentos de maior proximidade psíquica que oportunizavam confidências: Sabe o que me aconteceu esta semana? Encerrava-se o processo muitas vezes com um lamento, expressão de inusitada manifestação afetiva, provavelmente ativada pela sensibilidade à dor do desligamento: Já acabou? Não podemos fazer mais coisas? Ou mesmo só conversar?

\section{6- DISCUSSÃO}

Os resultados da avaliação psicodiagnóstica evidenciaram a preservação da capacidade intelectual - enquanto potencial, apesar da impossibilidade de utilização eficiente de todos os recursos cognitivos-racionais devido à invasão dos afetos, gerando acentuado sentimento de ineficiência ${ }^{16}$.

A avaliação dos aspectos emocionais, de uma forma geral, corroborou dados da literatura. O controle de impulsos dos pacientes é deficitário, podendo ocorrer episódios de auto e hetero agressividade, que colocam em risco tanto a própria integridade física como das pessoas do ambiente circundante. São pessoas com auto-estima rebaixada, que sofrem de intensa distorção da imagem corporal e sentimentos de desesperança ${ }^{6}$.

Essa configuração da organização afetiva, além de interferir no aproveitamento pleno dos recursos intelectuais, acaba prejudicando também a qualidade dos relacionamentos interpessoais, que são temidos e marcados pelo desejo de aprovação e extrema insegurança $\mathrm{a}^{16}$.

Devido aos intensos conflitos psicológicos experimentados, o relacionamento com as figuras parentais aparece permeado por marcada ambivalência emocional, prejudicando o processo de individuação e separação psicológica em relação aos pais, o que remete às questões de autonomia versus independência $^{2,16}$ como uma tarefa ainda não elaborada. A falta de elaboração dos conflitos psíquicos vivenciados na internalização das imagos parentais compromete a consolidação da identidade pessoal. Em decorrência da frágil estrutura egóica, em situações de maior vulnerabilidade psíquica os pacientes com transtornos alimentares recorrem a movimentos regressivos, na tentativa de se defenderem de vivências catastróficas de dispersão e perda dos limites da própria identidade. Isso se manifesta na tenacidade com que tentam preservar um padrão de relacionamento infantil com seus pais, agarrando-se a vínculos simbióticos - uma fusão eu-outro mortífera, que embaralha as fronteiras que definem a subjetividade - obstruindo os movimentos expansivos que normalmente impulsionam o adolescente na rota do crescimento emocional.

Apesar do marcado comprometimento psíquico detectado nos pacientes avaliados, durante o processo de avaliação psicodiagnóstica puderam ser observadas mudanças na forma como encaravam a doença e lidavam com o tratamento. É bastante freqüente que esses pacientes, no início do tratamento, acentuem uma tendência a negar a existência da doença, associando o transtorno alimentar a um estilo de vida excêntrico, porém válido, resultante de uma opção consciente a qual eles alçaram de forma deliberada, após examinarem várias alternativas. Abraçados aos seus sintomas sentem-se, inclusive, diferenciados da maioria dos mortais, uma vez que se consideram dotados de um estilo peculiar de levar a vida. Conseqüentemente, mostram-se em geral refratários ao tratamento ou, quando menos questionadores, apresentam uma aderência superficial ao esquema terapêutico proposto pela equipe.

Essa atitude de reserva em relação ao atendimento em geral reflete-se em uma atitude de desconfiança - que por vezes se traduz em manifestações de franca hostilidade e explosividade no relacionamento com algum profissional da equipe. Contudo, com a evolução do tratamento e o fortalecimento do vínculo com a equipe, tendem a reconhecer a necessidade de atenção especializada, embora uma parcela de pacientes ainda permaneça ambivalente em relação ao atendimento, guardando uma atitude defensiva de aparente indiferença e calculado distanciamento emocional.

Outro importante achado encontrado diz respeito à capacidade de vinculação com a psicóloga e uma crescente aproximação e abertura a experiências de intimidade emocional que foi ocorrendo ao longo do processo de avaliação, o que leva a refletir sobre a dificuldade - e não impossibilidade - de estabelecimento de vínculos interpessoais. Na medida em que a desconfiança foi abrandada e outras experiências emocionais abriram caminho para que se criassem 
condições para um relacionamento amistoso, pôde-se perceber uma maior permeabilidade aos afetos. O que sugere que esses pacientes podem se beneficiar de um tipo de psicoterapia que favoreça o exame do estado emocional vivenciado no aqui-e-agora, possibilitando assim uma experiência emocional corretiva.

\section{7- CONCLUSÃO}

A maior parte dos achados, vistos em seu conjunto, fornecem suporte empírico à hipótese de com- prometimento psíquico dos pacientes portadores de transtornos alimentares. O perfil psicológico delineado pelas avaliações realizadas revela dificuldades não apenas em termos de funcionamento dinâmico, como também de estrutura psíquica. Por outro lado, a despeito das limitações demonstradas no plano da organização psíquica dos pacientes com transtornos alimentares, os resultados evidenciaram também a possibilidade de vinculação e manutenção de laços afetivos, sugerindo a necessidade de suporte psicoterapêutico propiciador de amadurecimento emocional.

Oliveira EA, Santos MA. Psychological profile of patients who suffer from anorexia nervosa and bulimia nervosa: psychological assessment point of view. Medicina (Ribeirão Preto) 2006; 39 (3): 353-60.

ABSTRACT: The aim of this work is to present the psychological profile of patients who suffer from anorexia nervosa and bulimia nervosa in order to provide information for intervention planning by the interdisciplinary team. 29 psychodiagnostic assessments were carried out with 24 women and 5 men. The used tools were projective techniques, intellectual level test, standardized scales and clinical interview. The outcomes of this research were: denial of the illness and consequently of the necessity of treatment stood out in the interviews. As to the outcome provided by the others tools it was seen, by and large, that the intellectual resources are within the expected average but some emotional disturbs of patients who suffer from eating disorders showed up, despite the bond possibility, which proves the need of psychotherapeutically support. ders.

Keywords: Anorexia Nervosa. Bulimia Nervosa. Psychological Assessment. Eating Disor-

\section{REFERÊNCIAS}

1 - Doyle J, Bryant-Waugh R. Epidemiology. In: Lask B, BryantWaugh R, eds. Anorexia nervosa and related eating disorders in childhood and adolescence. $2^{\text {th }}$ ed. East Sussex: Psychology Press; 2000. p. 41-61.

2 - Lawrence M. A experiência anoréxica. São Paulo: Summus; 1991.

3 - Minuchin S, Rosman BL, Baker L. Psychosomatic families: anorexia nervosa in context. Cambridge, MA: Harvard University Press; 1978.

4 - Bruch H. Anorexia nervosa: theory and therapy. Am J Psychiatr 1982; 139 (12): 1531-8.

5 - Kreling DB, Santos MA. Anorexia nervosa: um relato de atendimento clínico realizado em contexto multidisciplinar. In: Santos MA, Simon, CP, Melo-Silva LL. Formação em Psicologia: processos clínicos. São Paulo: Vetor; 2005. p. 143-62.

6 - Abreu CN, Filho Cangelli R. Anorexia nervosa e bulimia nervosa: abordagem cognitiva-construtivista de psicoterapia. Rev Psiquiatr Clin 2004; 31(4): 177-83.

7 - Ocampo MLS, Arzeno MEG, Picollo EG. O processo psicodiagnóstico e as técnicas projetivas. Trad. M Felzenszwalb. 8. ed. São Paulo: Martins Fontes; 1995.
8 - Biklen S, Bogdan R. Investigação qualitativa em educação: uma introdução à teoria e aos métodos. Porto: Porto Editora; 1994.

9 - Raven JC. Manual dos Testes das Matrizes Progressivas Escala Geral. Trad. F. Campos. Rio de Janeiro: CEPA, s/d.

10 - França-e-Silva E. O Teste de Apercepção Temática de Murray (TAT) na cultura brasileira: manual de aplicação e interpretação. São Paulo: Fundação Getúlio Vargas/ISOP; 1984.

11 - Nijamikin GC, Braude MG. O Questionário Desiderativo. Trad. LS de La Plata Cury Tardivo. São Paulo: Vetor; 2000.

12 - Pasian SR. O psicodiagnóstico de Rorschach em adultos: atlas, normas e reflexões. São Paulo: Casa do Psicólogo; 2000.

13 - Villemor-Amaral F. Pirâmides Coloridas de Pfister. Rio de Janeiro: CEPA; 1978.

14 - Hammer EF, org. Aplicações clínicas dos desenhos projetivos. Trad. E Nick. Rio de Janeiro: Interamericana; 1981.

15 - Triviños ANS. Introdução à pesquisa em Ciências Sociais: a pesquisa qualitativa em educação. São Paulo: Atlas; 1992.

16 - Bruch $\mathrm{H}$. The golden cage: the enigma of anorexia nervosa. Cambridge, MA: Harvard University Press; 1978. 